\title{
Eigenvalue Inequalities and Schubert Calculus
}

\author{
Uwe Helmke \\ University of Regensburg \\ 8400 Regensburg \\ GERMANY
}

\author{
Joachim Rosenthal ${ }^{1}$ \\ University of Notre Dame \\ Notre Dame, IN 46556 \\ USA
}

April 1994.

\begin{abstract}
Using techniques from algebraic topology we derive linear inequalities which relate the spectrum of a set of Hermitian matrices $A_{1}, \ldots, A_{r} \in \mathbb{C}^{n \times n}$ with the spectrum of the sum $A_{1}+\cdots+A_{r}$. These extend eigenvalue inequalities due to Freede-Thompson and Horn for sums of eigenvalues of two Hermitian matrices.
\end{abstract}

\section{Introduction}

Consider real $n \times n$ diagonal matrices $D_{1}, \ldots, D_{r}$ with diagonal elements $\lambda_{1}\left(D_{l}\right) \geq$ $\lambda_{2}\left(D_{l}\right) \geq \ldots \geq \lambda_{n}\left(D_{l}\right), l=1, \ldots, r$. In this paper we are concerned with geometric properties of the set of possible spectrums of the matrices

$$
\left\{\sum_{l=1}^{r} U_{l}^{*} D_{l} U_{l} \mid U_{l} \text { are unitary }\right\}
$$

Equivalently we are interested in the following question:

Given Hermitian matrices $A_{1}, \ldots, A_{r} \in \mathbb{C}^{n \times n}$ each with a fixed spectrum $\lambda_{1}\left(A_{l}\right) \geq$ $\ldots \geq \lambda_{n}\left(A_{l}\right), l=1, \ldots, r$ and arbitrary else. Is it possible to find then linear inequalities which describe the possible spectrum of the matrix $A_{1}+\cdots+A_{r}$ ?

For $r=1$ this question is of course trivial. For $r=2$ the question is classical and very well studied (compare with $[5,7,8,9,15,16,17,18]$ ).

An early example of an eigenvalue inequality for a sum of two Hermitian matrices is that of Weyl [18]. A generalization of the Weyl inequalities to $k$-fold partial sums of eigenvalues of Hermitian matrices $A, B$ and $A+B$ is due to Freede and Thompson

\footnotetext{
${ }^{1}$ Supported in part by NSF grant DMS-9201263.
} 
[17]. Still more general is the class of eigenvalue inequalities described by Horn [7] for sums of two eigenvalues.

In this paper we will present a systematic geometric approach to obtain such eigenvalue inequalities. Although our main results are in the case of two matrices, where $r=2$, the approach works equally well in the case of $r$-fold sums $A_{1}+\cdots+A_{r}$ of Hermitian matrices $A_{1}, \ldots, A_{r}$. Our interest in this problem originates in the observation by Thompson $[15,16]$ who indicates that most of the known inequalities for the case $r=2$ can be derived using methods from algebraic topology, i.e. by the Schubert calculus of complex Grassmann manifolds. As this topological approach is described only in a rudimentary form in $[15,16]$ we first present a rigorous development of the Schubert calculus technique towards eigenvalue inequalities. We then show that it is also possible to derive with the same method a large set of inequalities for the case $r>2$ as well.

The algebraic topology approach to solving inverse eigenvalue problems is by no means limited to the task of finding eigenvalue inequalities for sums of Hermitian matrices. In fact, the technique has been already successfully applied to solve an outstanding inverse eigenvalue problem arising in control theory, i.e. the pole placement problem for multivariable linear systems by static output feedback. For this we refer to e.g. $[2,12]$.

The paper is structured as follows: In the next section the minmax principles of Wielandt and Hersch-Zwahlen are reviewed, which characterize in geometric terms partial sums of eigenvalues of a Hermitian matrix. In Section 3 we review the relevant results from the Schubert calculus of Grassmann manifolds. In Section 4 we apply the technique and state the main results. In Section 5 we show how the inequalities of Weyl [18], [9] and Freede-Thompson [17] follow from the main theorem. In the last section we describe a large set of nonzero products in the cohomology ring $H^{*}\left(G_{k}\left(\mathbb{C}^{n}\right), \mathcal{Z}\right)$ of the Grassmann manifold, leading to a new class of inequalities for sums of eigenvalues of Hermitian matrices $A_{1}, \ldots, A_{r}$.

\section{Min-Max-Principles and Rayleigh quotients}

Let $A \in \mathbb{C}^{n \times n}$ be a complex Hermitian matrix with eigenvalues

$$
\lambda_{1}(A) \geq \lambda_{2}(A) \geq \ldots \geq \lambda_{n}(A) .
$$

The classical Courant-Fischer minmax principle then asserts that (compare e.g. [1]):

Theorem 2.1 For $1 \leq i \leq n$ :

$$
\begin{aligned}
\lambda_{i}(A) & =\max _{\operatorname{dim} V=i} \min _{\substack{x \in V \\
\|x\|=1}} \operatorname{tr}\left(A x x^{*}\right) \\
& =\min _{\operatorname{dim} W=n-i+1} \max _{\substack{x \in W \\
\|x\|=1}} \operatorname{tr}\left(A x x^{*}\right)
\end{aligned}
$$


A more general version of the minmax principle is due to Wielandt [19] and HerschZwahlen [5] and characterizes partial sums of eigenvalues via flags of subspaces of $\mathbb{C}^{n}$. To state their result we first recall some basic notions and definitions from geometry:

The complex projective space $\mathbb{C} \mathbb{P}^{n}$ is defined as the set of all one-dimensional complex subspaces of $\mathbb{C}^{n+1}$, i.e. as the set of all complex lines passing through the origin $0 \in \mathbb{C}^{n+1}$. More generally, the complex Grassmann manifold $G_{k}\left(\mathbb{C}^{n}\right)$ is defined as the set of all $k$-dimensional complex linear subspaces of $\mathbb{C}^{n}$. In particular for $k=1$ one has the complex projective space $G_{1}\left(\mathbb{C}^{n}\right)=\mathbb{C P}^{n-1}$. The Grassmannian is a smooth, compact manifold of real dimension $2 k(n-k)$.

Equivalently, the Grassmannian $G_{k}\left(\mathbb{C}^{n}\right)$ may be defined as the set of all Hermitian projection operators $P: \mathbb{C}^{n} \rightarrow \mathbb{C}^{n}$ of rank $k$. A Hermitian projection operator of $\mathbb{C}^{n}$ is a Hermitian matrix $P \in \mathbb{C}^{n \times n}$ satisfying

$$
P^{*}=P, \quad P^{2}=P, \quad \text { and } \operatorname{rank} P=k .
$$

For any $k$-dimensional complex linear subspace $L \subset \mathbb{C}^{n}$ let $P_{L}: \mathbb{C}^{n} \rightarrow \mathbb{C}^{n}$ be the uniquely determined projection operator satisfying

$$
\operatorname{im}\left(P_{L}\right)=L, \quad \operatorname{ker}\left(P_{L}\right)=L^{\perp},
$$

where $L^{\perp}$ denotes the orthogonal complement of $L$ in $\mathbb{C}^{n}$ with respect of the standard Hermitian inner product. Thus $P_{L}$ is the orthogonal projection of $\mathbb{C}^{n}$ onto $L$ along $L^{\perp}$. If $X \in \mathbb{C}^{n \times k}$ is any full rank matrix whose columns form a basis of $L$, then one has

$$
P_{L}=X\left(X^{*} X\right)^{-1} X^{*} .
$$

Conversely, for any full rank matrix $X \in \mathbb{C}^{n \times k}$, the operator defined by (2.6) is a rank $k$ Hermitian projection operator on $\mathbb{C}^{n}$. Thus the map $L \mapsto P_{L}$ is a bijection of $G_{k}\left(\mathbb{C}^{n}\right)$ onto the set

$$
\left\{P \in \mathbb{C}^{n \times n} \mid P^{*}=P, \quad P^{2}=P, \quad \text { and } \operatorname{rank} P=k\right\} .
$$

Given any $k$-dimensional linear subspace $L \subset \mathbb{C}^{n}$ let $P_{L}: \mathbb{C}^{n} \rightarrow \mathbb{C}^{n}$ denote the associated Hermitian projection operator. We then define

$$
\begin{aligned}
\operatorname{tr}\left(\left.A\right|_{L}\right) & :=\operatorname{tr}\left(P_{L} A P_{L}\right) \\
& =\operatorname{tr}\left(A P_{L}\right) \\
& =\operatorname{tr}\left(A X\left(X^{*} X\right)^{-1} X^{*}\right)
\end{aligned}
$$

where $X \in \mathbb{C}^{n \times k}$ is any full rank matrix whose columns form a basis of $L$. Note that $\operatorname{tr}\left(\left.A\right|_{L}\right)$ is the trace of a Hermitian operator and therefore a real number. 
Definition 2.2 The smooth map

$$
\begin{aligned}
R_{A}: \quad G_{k}\left(\mathbb{C}^{n}\right) & \longrightarrow \mathbb{R} \\
L & \longmapsto \operatorname{tr}\left(\left.A\right|_{L}\right)
\end{aligned}
$$

is called the Rayleigh quotient of $A$ on $G_{k}\left(\mathbb{C}^{n}\right)$.

If $k=1$ the map $R_{A}$ coincides with the classical Rayleigh quotient

$$
R_{A}(x)=\frac{<A x, x>}{<x, x>} .
$$

The extremal principles for the partial sums of eigenvalues of a Hermitian matrix $A$ of Wielandt, Hersch-Zwahlen and Riddel are now stated as follows:

Theorem 2.3 (Wielandt [19]) For $1 \leq i_{1}<\ldots<i_{k} \leq n$ :

$$
\lambda_{i_{1}}(A)+\cdots+\lambda_{i_{k}}(A)=\max _{\substack{V_{1} \subset \ldots \subset V_{k} \\ \operatorname{dim} V_{j}=i_{j}}} \quad \min _{\substack{L \in G_{k}\left(\mathbb{C}^{n}\right) \\ \operatorname{dim}\left(L \cap V_{j}\right) \geq j}} \operatorname{tr}\left(\left.A\right|_{L}\right)
$$

In particular, for $k=1$, Theorem 2.3 specializes to the Courant-Fischer minmax principle as formulated in Theorem 2.1.

Remark 2.4 It can be shown (see Bhatia [1, page 43]) that the maximal value of (2.10) is assumed at a "partial flag of eigenspaces", i.e. at a flag $\left(V_{1}, \ldots, V_{k}\right)$ having the property that

$$
\operatorname{dim}\left(V_{j}\right)=i_{j} \text { and } V_{j} \subset \operatorname{ker}\left(\lambda_{1} I-A\right) \oplus \cdots \oplus \operatorname{ker}\left(\lambda_{i_{j}} I-A\right) \text {, for } j=1, \ldots, k \text {. }
$$

We conclude this section with the following result from Hersch-Zwahlen [5]:

Theorem 2.5 Let $A$ be a Hermitian matrix with eigenvalues $\lambda_{1}(A) \geq \ldots \geq \lambda_{n}(A)$ and a corresponding orthogonal set of eigenvectors $v_{1}, \ldots, v_{n}$. Denote with

$$
V_{m}:=\operatorname{span}\left(v_{1}, \ldots, v_{m}\right), m=1, \ldots, n .
$$

Let $1 \leq i_{1}<\ldots<i_{k} \leq n$. Then one has:

$$
\lambda_{i_{1}}(A)+\cdots+\lambda_{i_{k}}(A)=\min _{L \in G_{k}\left(\mathbb{C}^{n}\right)}\left\{\operatorname{tr}\left(\left.A\right|_{L}\right) \mid \operatorname{dim}\left(L \cap V_{i_{j}}\right) \geq j, \quad j=1, \ldots, k\right\} .
$$

Thus the result of Hersch-Zwahlen just says that the sum of eigenvalues $\lambda_{i_{1}}(A)+$ $\cdots+\lambda_{i_{k}}(A)$ is characterized as the minimal value of the trace function $\operatorname{tr}\left(\left.A\right|_{L}\right)$ when evaluated on a Schubert subvariety of $G_{k}\left(\mathbb{C}^{n}\right)$. 


\section{Schubert Calculus}

Consider again the Grassmann manifold $G_{k}\left(\mathbb{C}^{n}\right)$ consisting of $k$-dimensional linear subspaces of the vector space $\mathbb{C}^{n}$. Using the Plücker embedding $G_{k}\left(\mathbb{C}^{n}\right)$ can be embedded into the projective space $\mathbb{C P}^{N}$ of dimension $N=\frac{n !}{k !(n-k) !}-1$. Under this embedding $G_{k}\left(\mathbb{C}^{n}\right)$ is a projective variety described by a famous set of quadratic relations (see e.g. [4]).

Definition 3.1 A flag $\mathcal{F}$ is a sequence of nested subspaces

$$
\{0\} \subset V_{1} \subset V_{2} \subset \ldots \subset V_{n}=\mathbb{C}^{n}
$$

where we assume that $\operatorname{dim} V_{i}=i$ for $i=1, \ldots, n$.

Let $\underline{i}=\left(i_{1}, \ldots, i_{k}\right)$ denote a sequence of numbers having the property that

$$
1 \leq i_{1}<\ldots<i_{k} \leq n
$$

Definition 3.2 For each flag $\mathcal{F}$ and each multiindex $\underline{i}$ define:

$$
C(\underline{i} ; \mathcal{F}):=\left\{W \in G_{k}\left(\mathbb{C}^{n}\right) \mid \operatorname{dim}\left(W \bigcap V_{i_{s}}\right)=s\right\}
$$

is called a Schubert cell and

$$
S(\underline{i} ; \mathcal{F}):=\left\{W \in G_{k}\left(\mathbb{C}^{n}\right) \mid \operatorname{dim}\left(W \bigcap V_{i_{s}}\right) \geq s\right\}
$$

is called a Schubert variety.

We emphasize that the Schubert cell $C(\underline{i} ; \mathcal{F})$ is indeed a cell, i.e. isomorphic to the affine space $\mathbb{C}^{N}$ where $N:=\sum_{j=1}^{k} i_{j}-j$ is the dimension of the cell $C(\underline{i} ; \mathcal{F})$. (Compare with [4].) Moreover the Zariski closure of the cell $C(\underline{i} ; \mathcal{F})$ is the variety $S(\underline{i} ; \mathcal{F})$, which is a projective algebraic subvariety of $G_{k}\left(\mathbb{C}^{n}\right)$.

The following results are well known and we refer e.g. to $[3,4]$.

Theorem 3.3 For every fixed flag $\mathcal{F}$ the Schubert cells $C(\underline{i} ; \mathcal{F})$ decompose the Grassmann variety $G_{k}\left(\mathbb{C}^{n}\right)$ into a finite cellular $C W$-complex. The integral homology $H_{2 m}\left(G_{k}\left(\mathbb{C}^{n}\right), \mathcal{Z}\right)$ has no torsion and is freely generated by the fundamental classes of the Schubert varieties $S(\underline{i} ; \mathcal{F})$ of real dimension $2 m$.

Consider a fixed Schubert variety $S(\underline{i} ; \mathcal{F})$. Its homology class is independent of the choice of the flag $\mathcal{F}$ and therefore depends only on the numbers $i_{1}, \ldots, i_{k}$. We will use the symbol $\left(i_{1}, \ldots, i_{k}\right)$ to denote this homology class. The Poincaré-dual of the class $\left(i_{1}, \ldots, i_{k}\right)$ will be denoted by

$$
\left\{\mu_{1}, \ldots, \mu_{k}\right\}:=\left\{n-k-i_{1}+1, n-k-i_{2}+2, \ldots, n-i_{k}\right\} \in H^{*}\left(G_{k}\left(\mathbb{C}^{n}\right), \mathcal{Z}\right) .
$$


At this point we want to mention that our notation was already used by Schubert (compare with the book of Fulton [3, page 271]) and is slightly different to the one used in $[4,6]$. The cohomology ring

$$
H^{*}\left(G_{k}\left(\mathbb{C}^{n}\right), \mathcal{Z}\right):=\bigoplus_{m=0}^{k(n-k)} H^{2 m}\left(G_{k}\left(\mathbb{C}^{n}\right), \mathcal{Z}\right)
$$

has in a natural way the structure of a graded ring. From Poincaré-duality and Theorem 3.3 it follows in particular that each graded component $H^{2 m}\left(G_{k}\left(\mathbb{C}^{n}\right), \mathcal{Z}\right)$ is a free $\mathcal{Z}$-module with basis the set of Schubert cocycles $\left\{\mu_{1}, \ldots, \mu_{k}\right\}$ where $n-k \geq$ $\mu_{1} \geq \ldots \geq \mu_{k} \geq 0$ and $\sum_{j=1}^{k} \mu_{j}=m$.

Before we describe the multiplicative structure of this ring we formulate the following proposition which establishes the crucial link between geometric intersection properties of Schubert varieties and algebraic properties of the ring $H^{*}\left(G_{k}\left(\mathbb{C}^{n}\right), \mathcal{Z}\right)$. A proof of this as well as more general theorems can be found e.g. in $[3,4]$.

Proposition 3.4 Consider $r$ Schubert varieties $S\left(\underline{i}_{l} ; \mathcal{F}_{l}\right), l=1, \ldots, r$. If

$$
\prod_{l=1}^{r+1}\left\{n-k-i_{1 l}+1, \ldots, n-i_{k l}\right\} \neq 0,
$$

then the intersection

$$
\bigcap_{l=1}^{r} S\left(\underline{i}_{l} ; \mathcal{F}_{l}\right) \neq \emptyset .
$$

The multiplicative structure of $H^{*}\left(G_{k}\left(\mathbb{C}^{n}\right), \mathcal{Z}\right)$ is described by the classical formulas of Pieri and Giambelli. For this denote with

$$
\sigma_{j}:=\{j, 0, \ldots, \ldots, 0\} \quad j=1, \ldots, n-k .
$$

In fact $\sigma_{j}$ is the $j$-th Chern class of the universal (classifying) bundle over $G_{k}\left(\mathbb{C}^{n}\right)$.

In the following we describe the formulas of Pieri and Giambelli. Giambelli's formula expresses a general Schubert cocycle $\left\{\mu_{1}, \ldots, \mu_{k}\right\}$ as a polynomial in the special Schubert cocycle $\sigma_{j}$ and Pieri's formula expresses the product of a general Schubert cocycle with a special Schubert cocycle.

Pieri's formula:

$$
\left\{\mu_{1}, \ldots, \mu_{k}\right\} \cdot \sigma_{j}=\sum_{\substack{\mu_{i-1} \geq \nu_{i} \geq \mu_{i} \\ \sum_{i=1}^{k} \nu_{i}=\left(\sum_{i=1}^{k} \mu_{i}\right)+j}}\left\{\nu_{1}, \ldots, \nu_{k}\right\}
$$

Giambelli's formula:

$$
\left\{\mu_{1}, \ldots, \mu_{k}\right\}=\operatorname{det}\left(\sigma_{\mu_{i}+j-i}\right)=\operatorname{det}\left(\begin{array}{cccc}
\sigma_{\mu_{1}} & \sigma_{\mu_{1}+1} & \ldots & \sigma_{\mu_{1}+k-1} \\
\sigma_{\mu_{2}-1} & \sigma_{\mu_{2}} & & \vdots \\
\vdots & & \ddots & \vdots \\
\sigma_{\mu_{k}-k+1} & & \ldots & \sigma_{\mu_{k}}
\end{array}\right)
$$


Note that Giambelli's formula implies that the Chern classes $\sigma_{j}$ generate the ring $H^{*}\left(G_{k}\left(\mathbb{C}^{n}\right), \mathcal{Z}\right)$.

There is a deep relationship between the $\operatorname{ring} H^{*}\left(G_{k}\left(\mathbb{C}^{n}\right), \mathcal{Z}\right)$ and the ring of symmetric functions $\mathcal{Z}\left[x_{1}, \ldots, x_{k}\right]^{\mathcal{S}_{k}}$, where $\mathcal{S}_{k}$ denotes the group of permutations, acting on $k$ letters. To explain this relationship we consider a special set of symmetric functions called Schur functions. (See e.g. $[10,14])$. For this let $\mu:=\left(\mu_{1}, \ldots, \mu_{k}\right)$ and define

$$
s_{\mu}:=\frac{\operatorname{det}\left[x_{i}^{\mu_{j}+k-j}\right]}{\operatorname{det}\left[x_{i}^{k-j}\right]} ; \quad i, j=1, \ldots, k .
$$

Note that $s_{\mu}$ is the quotient of two alternating functions and therefore a symmetric function, called a Schur function. As explained in detail in [10] the set of Schur functions

$$
\left\{s_{\mu} \mid \mu_{1} \geq \mu_{2} \geq \ldots \geq \mu_{k}>0 \text { and } \sum \mu_{i}=q\right\}
$$

is an additive basis of the space of symmetric functions of degree $q$. As explained in $[6,12,14]$ one has a ring epimorphism

$$
\begin{aligned}
\psi: \mathcal{Z}\left[x_{1}, \ldots, x_{k}\right]^{\mathcal{S}_{k}} & \longrightarrow H^{*}\left(G_{k}\left(\mathbb{C}^{n}\right), \mathcal{Z}\right) \\
s_{\mu} & \longmapsto\left\{\mu_{1}, \ldots, \mu_{k}\right\}
\end{aligned}
$$

The kernel of this map has as an additive basis the set of Schur functions $s_{\mu}$ with $\mu_{1}>n-k$.

Using this epimorphism any calculation in the ring $H^{*}\left(G_{k}\left(\mathbb{C}^{n}\right), \mathcal{Z}\right)$ can be formally done in the $\operatorname{ring} \mathcal{Z}\left[x_{1}, \ldots, x_{k}\right]^{\mathcal{S}_{k}}$. In particular we want to mention the rule of Littlewood and Richardson which explains how to additively expand a product of Schur functions in terms of Schur functions:

Consider two Schur functions $s_{\mu}$ and $s_{\nu}$. The product $s_{\mu} s_{\nu}$ is a symmetric function of degree $\sum \mu_{i}+\sum \nu_{i}$ and has therefore an expansion in terms of Schur functions:

$$
s_{\mu} s_{\nu}=\sum_{\lambda} c_{\mu, \nu}^{\lambda} s_{\lambda}
$$

The appearing coordinates $c_{\mu, \nu}^{\lambda}$ are usually called the Littlewood Richardson coefficients $[10,13,14]$. In order to give a combinatorial characterization of those coefficients let $\mu=\left(\mu_{1}, \ldots, \mu_{k}\right)$ be a partition of $n$ representing the Schur function $s_{\mu}$. In other words we assume that $n-k \geq \mu_{1} \geq \mu_{2} \geq \ldots \geq \mu_{k} \geq 0$ and $\sum_{i=1}^{k} \mu_{i}=n$. If the integer $\mu_{i}$ is repeated $r_{i}$-times in the partition $\mu$, the abbreviated notation $\mu=\left(\mu_{1}^{r_{1}}, \ldots, \mu_{t}^{r_{t}}\right)$ will be used. The number $|\mu|:=\sum_{i=1}^{k} \mu_{i}$ is sometimes called the weight of the partition $\mu$ and the numbers $\mu_{i}$ are called the parts of the partition.

It is usual to present a partition by a left based array of boxes which has exactly $\mu_{i}$ boxes in the $i$-th row. Such an array is sometimes called a tableau. 
Example 3.5 Two partitions with corresponding diagrams are illustrated:
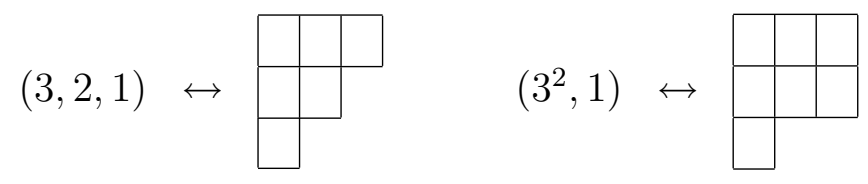

Let $\lambda=\left(\lambda_{1}, \ldots, \lambda_{k}\right)$ be a second partition. One writes $\lambda \geq \mu$ if $\lambda_{i} \geq \mu_{i}, i=1, \ldots, k$. If $\lambda \geq \mu$ one defines the skew tableau $\lambda / \mu$ as the tableau obtained from the tableau $\lambda$ by removing the first $\mu_{i}$ boxes in the row $i$ of the tableau $\lambda$.

Example 3.6 $\lambda=(5,4,2,2), \mu=(3,2,1)$ then $\lambda / \mu$ is given:

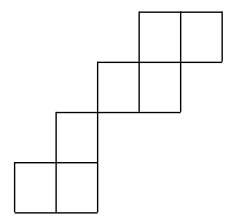

We are now in a position to formulate the theorem of Littlewood and Richardson. The following formulation as well as the subsequent example can be found in the article of Stanley [14].

Theorem 3.7 Let $s_{\mu}$ and $s_{\nu}$ be two Schur functions represented by two partitions $\mu, \nu$. Then the Littlewood Richardson coefficient $c_{\mu, \nu}^{\lambda}$ of $s_{\lambda}$ in the expansion of the product $s_{\mu} s_{\nu}$ is zero unless $\lambda \geq \mu$. In this case the coefficient is equal to the number of ways of inserting $\nu_{1}$ 1's, $\nu_{2}$ 2's, $\nu_{3}$ 3's, ... into the skew tableau $\lambda / \mu$ subject to the conditions:

1. The numbers are weakly increasing in each row and strictly increasing in each column.

2. If $\alpha_{1}, \alpha_{2}, \ldots$ is the set of numbers obtained when reading of the numbers inserted in $\lambda / \mu$ from right to left then for any $i, j$ the numbers of $i$ 's among $\alpha_{1}, \alpha_{2}, \ldots, \alpha_{j}$ is not less than the number $(i+1)$ 's among the numbers $\alpha_{1}, \alpha_{2}, \ldots, \alpha_{j}$.

The following example given in [14] illustrates the method:

Example 3.8 Let $\lambda=(5,4,2,2), \mu=(3,2,1)$ and $\nu=(4,2,1)$. Then the following skew diagrams $\lambda / \mu$ are the only ones which satisfy 1 . and 2. In particular the coefficient of $s_{\lambda}$ in the expansion of the product $s_{\mu} s_{\nu}$ is equal to 3 .
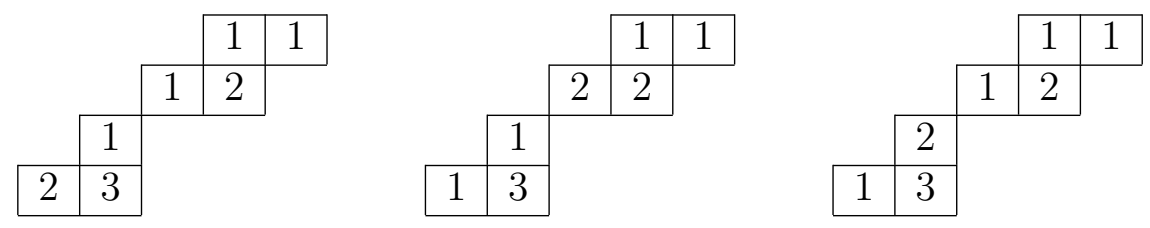
Using the Littlewood Richardson rule together with the description of the ring $H^{*}\left(G_{k}\left(\mathbb{C}^{n}\right), \mathcal{Z}\right)$ as given in $(3.12)$ we are in a position to multiply arbitrary cocycles in $H^{*}\left(G_{k}\left(\mathbb{C}^{n}\right), \mathcal{Z}\right)$. The following example illustrates the procedure:

Example 3.9 Consider the elements $\{3,2,0\}$ and $\{2,1,0\}$ in $H^{*}\left(G_{3}\left(\mathbb{C}^{6}\right), \mathcal{Z}\right)$. Then

$$
\begin{aligned}
\{3,2,0\}\{2,1,0\}= & \{5,3,0\}+\{5,2,1\}+\{4,4,0\}+2\{4,3,1\} \\
& +\{4,2,2\}+\{3,3,2\}
\end{aligned}
$$

We conclude this section with the Poincare duality theorem of cocycles. For this consider a cocycle $\left\{\mu_{1}, \ldots, \mu_{k}\right\}$. The dual cocycle in $H^{*}\left(G_{k}\left(\mathbb{C}^{n}\right), \mathcal{Z}\right)$ is defined as the cocycle $\nu:=\left\{n-k-\mu_{k}, \ldots, n-k-\mu_{1}\right\}$. Using this notation one has:

Theorem 3.10

$$
\left\{\mu_{1}, \ldots, \mu_{k}\right\}\left\{\nu_{1}, \ldots, \nu_{k}\right\}=\{n-k, \ldots, n-k\}
$$

Proof: Apply Theorem 3.7 of Littlewood and Richardson together with the description of $H^{*}\left(G_{k}\left(\mathbb{C}^{n}\right), \mathcal{Z}\right)$ induced by the representation (3.12).

\section{Main Results}

In order to derive our main result we will use the following simple lemma, the trivial proof of it is omitted.

Lemma 4.1 Suppose the eigenvalues of a Hermitian $n \times n$ matrix $A$ are ordered as $\lambda_{1}(A) \geq \ldots \geq \lambda_{n}(A)$. Then for any $1 \leq i_{1}<\ldots<i_{k} \leq n$ one has:

$$
\lambda_{i_{1}}(-A)+\cdots+\lambda_{i_{k}}(-A)=-\sum_{j=1}^{k} \lambda_{n-i_{j}+1}(A) .
$$

In the following we will consider Hermitian matrices $A_{1}, \ldots, A_{r+1} \in \mathbb{C}^{n \times n}$ with corresponding eigenvalues

$$
\lambda_{1}\left(A_{l}\right) \geq \ldots \geq \lambda_{n}\left(A_{l}\right), \quad l=1, \ldots, r+1
$$

and corresponding orthogonal sets of eigenvectors $v_{1 l}, \ldots, v_{n l}$. Assume that

$$
A_{r+1}=A_{1}+\cdots+A_{r} .
$$

For each Hermitian operator $A_{l}, l=1, \ldots, r+1$ construct a flag of eigenspaces

$$
\mathcal{F}_{l}: \quad\{0\} \subset V_{1 l} \subset V_{2 l} \subset \ldots \subset V_{n l}=\mathbb{C}^{n}
$$


defined through the property:

$$
V_{m l}:=\operatorname{span}\left(v_{1 l}, \ldots, v_{m l}\right) m=1, \ldots, n .
$$

The following result, which has been first proved by Thompson [17] for the case $r=2$, establishes the crucial relationship between matrix spectral inequalities and the Schubert calculus.

Lemma 4.2 Let $A_{1}, \ldots, A_{r}$ be complex Hermitian $n \times n$ matrices and denote with $\mathcal{F}_{1}, \ldots, \mathcal{F}_{r+1}$ the corresponding flags of eigenspaces defined by (4.5). Assume $A_{r+1}=$ $A_{1}+\cdots+A_{r}$. and let $\underline{i}_{l}=\left(i_{1 l}, \ldots, i_{k l}\right)$ be $r+1$ sequences of integers satisfying

$$
1 \leq i_{1 l}<\ldots<i_{k l} \leq n, \quad l=1, \ldots, r+1
$$

Suppose the intersection of the $r+1$ Schubert subvarieties of $G_{k}\left(\mathbb{C}^{n}\right)$ is nonempty, i.e.:

$$
S\left(\underline{i}_{1} ; \mathcal{F}_{1}\right) \bigcap \ldots \bigcap S\left(\underline{i}_{r+1} ; \mathcal{F}_{r+1}\right) \neq \emptyset .
$$

Then the following matrix eigenvalue inequalities hold:

$$
\begin{aligned}
& \sum_{j=1}^{k} \lambda_{n-i_{j, r+1}+1}\left(A_{1}+\cdots+A_{r}\right) \geq \sum_{l=1}^{r} \sum_{j=1}^{k} \lambda_{i_{j l}}\left(A_{l}\right) \\
& \sum_{j=1}^{k} \lambda_{i_{j, r+1}}\left(A_{1}+\cdots+A_{r}\right) \leq \sum_{l=1}^{r} \sum_{j=1}^{k} \lambda_{n-i_{j l}+1}\left(A_{l}\right) .
\end{aligned}
$$

Proof: Consider $L \in G_{k}\left(\mathbb{C}^{n}\right)$ with

$$
L \in \bigcap_{l=1}^{r+1} S\left(\underline{i}_{l} ; \mathcal{F}_{l}\right) \neq \emptyset .
$$

Then, by using the Hersch-Zwahlen extremal principle (Theorem 2.5) one has:

$$
\begin{aligned}
0= & \operatorname{tr}\left(\left.\left(A_{1}+\cdots+A_{r}-A_{r+1}\right)\right|_{L}\right) \\
= & \sum_{l=1}^{r} \operatorname{tr}\left(\left.A_{l}\right|_{L}\right)-\operatorname{tr}\left(\left.A_{r+1}\right|_{L}\right) \\
\geq & \sum_{l=1}^{r} \min \left\{\operatorname{tr}\left(\left.A_{l}\right|_{L}\right) \mid L \in S\left(\underline{i}_{l} ; \mathcal{F}_{l}\right)\right\} \\
& \quad+\min \left\{\operatorname{tr}\left(-\left.A_{r+1}\right|_{L}\right) \mid L \in S\left(\underline{i}_{r+1} ; \mathcal{F}_{r+1}\right)\right\} \\
= & \sum_{l=1}^{r} \sum_{j=1}^{k} \lambda_{i_{j l}}\left(A_{l}\right)+\sum_{j=1}^{k} \lambda_{i_{j, r+1}}\left(-A_{r+1}\right) .
\end{aligned}
$$


Thus by Lemma 4.1 one has:

$$
\sum_{j=1}^{k} \lambda_{n-i_{j, r+1}+1}\left(A_{r+1}\right) \geq \sum_{l=1}^{r} \sum_{j=1}^{k} \lambda_{i_{j l}}\left(A_{l}\right)
$$

which proves (4.8). The inequality (4.9) follows from (4.8) by replacing the matrices $A_{l}$ by $-A_{l}, l=1, \ldots, r+1$ and using Lemma 4.1. This completes the proof.

In general it will be difficult to verify the intersection property (4.7) as it assumes the knowledge of the eigenspaces of $A_{1}, \ldots, A_{r}$ and of $A_{r+1}=A_{1}+\cdots+A_{r}$. By combining Lemma 4.2 with the intersection theoretic result of Proposition 3.4 we obtain a result with a more easily verifiable hypothesis.

Theorem 4.3 Let $\underline{i}_{l}=\left(i_{1 l}, \ldots, i_{k l}\right)$ be $r+1$ sequences of integers satisfying

$$
1 \leq i_{1 l}<\ldots<i_{k l} \leq n, \quad l=1, \ldots, r+1
$$

Let $\left\{n-k-i_{1 l}+1, \ldots, n-i_{k l}\right\} \in H^{*}\left(G_{k}\left(\mathbb{C}^{n}\right), \mathcal{Z}\right)$ denote the Schubert cocycle that is the Poincaré dual of the fundamental homology class of the Schubert variety $S\left(\underline{i}_{l} ; \mathcal{F}_{l}\right)$ for $l=1, \ldots, r+1$. If the $(r+1)$-fold product of the Schubert cocycles in $H^{*}\left(G_{k}\left(\mathbb{C}^{n}\right), \mathcal{Z}\right)$

$$
\prod_{l=1}^{r+1}\left\{n-k-i_{1 l}+1, \ldots, n-i_{k l}\right\} \neq 0,
$$

then the eigenvalue inequality (4.8) and (4.9) holds for any set of Hermitian matrices $A_{1}, \ldots, A_{r} \in \mathbb{C}^{n \times n}$.

Proof: Immediate consequence of Lemma 4.2 and Proposition 3.4.

Corollary 4.4 Let $\underline{i}:=\left(i_{1}, \ldots, i_{k}\right), \underline{j}:=\left(j_{1}, \ldots, j_{k}\right), \underline{p}:=\left(p_{1}, \ldots, p_{k}\right)$, be sequences satisfying $1 \leq i_{1}<\ldots<i_{k} \leq n, 1 \leq \bar{j}_{1}<\ldots<j_{k} \leq n$ and $1 \leq p_{1}<\ldots<p_{k} \leq n$. If the triple product

$$
\left\{n-k-i_{1}+1, \ldots, n-i_{k}\right\}\left\{n-k-j_{1}+1, \ldots, n-j_{k}\right\}\left\{n-k-p_{1}+1, \ldots, n-p_{k}\right\} \neq 0,
$$

is nonzero then for any pair of complex Hermitian matrices $A, B \in \mathbb{C}^{n \times n}$ the following eigenvalue inequalities hold:

$$
\begin{aligned}
\sum_{\nu=1}^{k} \lambda_{n-p_{\nu}+1}(A+B) & \geq \sum_{\nu=1}^{k} \lambda_{i_{\nu}}(A)+\sum_{\nu=1}^{k} \lambda_{j_{\nu}}(B) \\
\sum_{\nu=1}^{k} \lambda_{p_{\nu}}(A+B) & \leq \sum_{\nu=1}^{k} \lambda_{n-i_{\nu}+1}(A)+\sum_{\nu=1}^{k} \lambda_{n-j_{\nu}+1}(B) .
\end{aligned}
$$


We conclude this section with a simple example.

Example 4.5 In $H^{*}\left(G_{2}\left(\mathbb{C}^{4}\right), \mathcal{Z}\right)$ the following nonzero products exist:

$$
\begin{aligned}
\{1,0\}\{1,0\}\{2,0\} & =\{2,2\} \\
\{1,0\}\{1,0\}\{1,1\} & =\{2,2\} \\
\{1,0\}\{1,0\}\{1,0\}\{1,0\} & =2\{2,2\} .
\end{aligned}
$$

By Theorem 4.3 and Corollary 4.4 the following eigenvalue inequalities hold for arbitrary $4 \times 4$ Hermitian matrices:

$$
\begin{gathered}
\lambda_{1}(A+B)+\lambda_{4}(A+B) \leq \lambda_{1}(A)+\lambda_{3}(A)+\lambda_{1}(B)+\lambda_{3}(B) \\
\lambda_{2}(A+B)+\lambda_{3}(A+B) \leq \lambda_{1}(A)+\lambda_{3}(A)+\lambda_{1}(B)+\lambda_{3}(B), \\
\lambda_{2}(A+B+C)+\lambda_{4}(A+B+C) \leq \lambda_{1}(A)+\lambda_{3}(A)+\lambda_{1}(B)+\lambda_{3}(B)+\lambda_{1}(C)+\lambda_{3}(C) .
\end{gathered}
$$

\section{Corollaries and Consequences}

We apply the preceding results to verify some classical eigenvalue inequalities. The first inequality is given in [18].

\subsection{Weyl inequality [18]:}

For any indices $1 \leq i, j \leq n$ with $1 \leq i+j-1 \leq n$ and any Hermitian matrices $A, B \in \mathbb{C}^{n \times n}$ one has:

$$
\lambda_{i+j-1}(A+B) \leq \lambda_{i}(A)+\lambda_{j}(B) .
$$

Proof: Here $k=1, G_{1}\left(\mathbb{C}^{n}\right)=\mathbb{C} \mathbb{P}^{n-1}$ and $\left.H^{*}\left(\mathbb{C P}^{n-1}\right), \mathcal{Z}\right)=\mathcal{Z}[x] /\left(x^{n}\right)$ is a truncated polynomial ring. Using this classical description of the cohomology ring of the projective space, the Schubert cocycles are

$$
\{i\}=x^{i}, \quad i=0, \ldots, n-1 .
$$

Let $i_{1}, j_{1}$ and $p_{1}$ defined by:

$$
i_{1}:=n-i+1, \quad j_{1}:=n-j+1, \quad p_{1}:=i+j-1 .
$$

Then (4.18) reduces to

$$
\{i-1\}\{j-1\}\{n-i-j+1\}=\{n-1\} .
$$

But since $x^{n-1}$ generates $\left.H^{2(n-1)}\left(\mathbb{C P}^{n-1}\right), \mathcal{Z}\right) \equiv \mathcal{Z}$ one has $\{n-1\} \neq 0$. Thus the Weyl inequality follows immediately from Corollary 4.4 . 


\subsection{Lidskii inequality:}

For $1 \leq a_{1}<\ldots<a_{k} \leq n$ and for any Hermitian matrices $A, B \in \mathbb{C}^{n \times n}$ one has the matrix eigenvalue inequality:

$$
\sum_{j=1}^{k} \lambda_{a_{j}}(A+B) \leq \sum_{j=1}^{k} \lambda_{a_{j}}(A)+\sum_{j=1}^{k} \lambda_{j}(B) .
$$

Proof: Consider $\underline{i}:=\left(n-a_{k}+1, \ldots, n-a_{1}+1\right), j:=(n-k+1, \ldots, n), p:=\left(a_{1}, \ldots, a_{k}\right)$. Then the product in condition (4.18) of Corollary 4.4 is given by

$$
\left\{a_{k}-k, \ldots, a_{1}-1\right\}\{0, \ldots, 0\}\left\{n-k-a_{1}+1, \ldots, n-a_{k}\right\} .
$$

Since $\{0, \ldots, 0\}=1 \in H^{*}\left(G_{k}\left(\mathbb{C}^{n}\right), \mathcal{Z}\right)$ and $\left\{n-k-a_{1}+1, \ldots, n-a_{k}\right\}$ is Poincaré dual to $\left\{a_{k}-k, \ldots, a_{1}-1\right\}$ the above triple product is equal to $\{n-k, \ldots, n-k\}$ and hence nonzero. This completes the proof of the Lidskii inequality.

Thus both the Weyl and the Lidskii inequality are direct consequences of the Poincaré duality of the projective space $\mathbb{C P}^{n-1}$ and of the Grassmannian $G_{k}\left(\mathbb{C}^{n}\right)$ respectively. A proof of the next inequality requires a more subtle topological argument.

\subsection{Freede-Thompson inequality [17]:}

For any $1 \leq a_{1}<\ldots<a_{k} \leq n, 1 \leq b_{1}<\ldots<b_{k} \leq n$ with $a_{k}+b_{k}-k \leq n$ and Hermitian matrices $A, B \in \mathbb{C}^{n \times n}$ one has:

$$
\sum_{\nu=1}^{k} \lambda_{a_{\nu}+b_{\nu}-\nu}(A+B) \leq \sum_{\nu=1}^{k} \lambda_{a_{\nu}}(A)+\sum_{\nu=1}^{k} \lambda_{b_{\nu}}(B) .
$$

Proof: Consider $\underline{i}:=\left(n-a_{k}+1, \ldots, n-a_{1}+1\right), j:=\left(n-b_{k}+1, \ldots, n-b_{1}+1\right)$, $\underline{p}:=\left(a_{1}+b_{1}-1, \ldots, a_{k}+b_{k}-k\right)$. Then the product in condition (4.18) of Corollary 4.4 is given by

$$
\left\{a_{k}-k, \ldots, a_{1}-1\right\}\left\{b_{k}-k, \ldots, b_{1}-1\right\}\left\{n-k-a_{1}-b_{1}+2, \ldots, n+k-a_{k}-b_{k}\right\} .
$$

By assumption one has $a_{k}+b_{k}-2 k \leq n-k$. From the Littlewood Richardson rule it follows that the product of the first two factors is of the form:

$$
\left\{a_{k}-k, \ldots, a_{1}-1\right\}\left\{b_{k}-k, \ldots, b_{1}-1\right\}=\left\{a_{k}+b_{k}-2 k, \ldots, a_{1}+b_{1}-2\right\}+\sum_{\lambda} c_{\mu, \nu}^{\lambda}\{\lambda\},
$$

where $c_{\mu, \nu}^{\lambda}$ are again the Littlewood Richardson coefficients and the sum is taken over all partitions $\lambda, \lambda \neq\left\{a_{k}+b_{k}-2 k, \ldots, a_{1}+b_{1}-2\right\}$. Now the result follows from the observation that the cocycle $\left\{a_{k}+b_{k}-2 k, \ldots, a_{1}+b_{1}-2\right\}$ is (compare with Theorem 3.10) dual to the cocycle $\left\{n-k-a_{1}-b_{1}+2, \ldots, n+k-a_{k}-b_{k}\right\}$, i.e. the product (5.8) is nonzero and Theorem 4.3 applies. 


\section{$6 \quad$ Nonzero Products in $H^{*}\left(G_{k}\left(\mathbb{C}^{n}\right), \mathcal{Z}\right)$}

It is a consequence of Theorem 4.3 that any nonzero product in $H^{*}\left(G_{k}\left(\mathbb{C}^{n}\right), \mathcal{Z}\right)$ implies an eigenvalue inequality of the form (4.8) and an inequality of the form (4.9). In this section we describe a large class of nonzero products. In particular we will describe all maximal nonzero products in $H^{*}\left(G_{2}\left(\mathbb{C}^{n}\right), \mathcal{Z}\right)$ and we will describe all maximal nonzero products in $H^{*}\left(G_{k}\left(\mathbb{C}^{n}\right), \mathcal{Z}\right)$ consisting of 3 factors. The following lemmas prepare for those results.

Lemma 6.1 Assume $\mu:=\left\{\mu_{1}, \ldots, \mu_{k}\right\}$ and $\nu:=\left\{\nu_{1}, \ldots, \nu_{k}\right\}$ are two cocycles in $H^{*}\left(G_{k}\left(\mathbb{C}^{n}\right), \mathcal{Z}\right)$ which are complimentary in dimension, i.e. there weights satisfy $|\mu|+|\nu|=k(n-k)$. Then $\mu \nu \neq 0$ if, and only if $\mu$ and $\nu$ are dual to each other, i.e. $\nu=\left\{n-k-\mu_{k}, \ldots, n-k-\mu_{1}\right\}$.

Proof: See also [4, p.198] for a different proof based on Poincaré-duality. From the description of $H^{*}\left(G_{k}\left(\mathbb{C}^{n}\right), \mathcal{Z}\right)$ in $(3.12)$ it is clear that $\mu \nu \neq 0$ exactly when the coefficient of $\left\{(n-k)^{k}\right\}=\{n-k, \ldots, n-k\}$ in the expansion $\mu \nu$ is nonzero. Applying the rule of Littlewood and Richardson to the skew tableau $(n-k)^{k} / \mu$ one verifies that there is only one possibility to fill this tableau with $\nu_{1} 1$ 's, $\nu_{2} 2$ 's, .., $\nu_{k} \mathrm{k}$ 's, and in this case one necessarily has $\nu_{1}=n-k-\mu_{k}, \ldots, \nu_{k}=n-k-\mu_{1}$.

Lemma 6.2 Assume $\mu_{l}=\left\{\mu_{1 l}, \ldots, \mu_{k l}\right\}, l=1, \ldots, r$, are cocycles with $\sum_{l=1}^{r} \mu_{1 l} \leq$ $n-k$. Then the following identity holds in $H^{*}\left(G_{k}\left(\mathbb{C}^{n}\right), \mathcal{Z}\right)$ :

$$
\left\{n-k-\sum_{l=1}^{r} \mu_{k l}, \ldots, n-k-\sum_{l=1}^{r} \mu_{1 l}\right\} \prod_{l=1}^{r}\left\{\mu_{1 l}, \ldots, \mu_{k l}\right\}=\{n-k, \ldots, n-k\} .
$$

Proof: Using inductively Littlewood Richardson's rule it follows that

$$
\prod_{l=1}^{r}\left\{\mu_{1 l}, \ldots, \mu_{k l}\right\}=\left\{\sum_{l=1}^{r} \mu_{1 l}, \ldots, \sum_{l=1}^{r} \mu_{k l}\right\}+\sum_{\mu} c_{\mu}\left\{\mu_{1}, \ldots, \mu_{k}\right\} .
$$

(Compare with (5.9)). Because $\left\{n-k-\sum_{l=1}^{r} \mu_{k l}, \ldots, n-k-\sum_{l=1}^{r} \mu_{1 l}\right\}$ is the Poincaré dual of the first term after the equality sign the result follows from the previous Lemma.

In the next Lemma we will identify the Schubert symbol $\left\{x_{1}, x_{2}\right\} \in H^{*}\left(G_{2}\left(\mathbb{C}^{n}\right), \mathcal{Z}\right)$ with zero for $x_{1}>n-2$.

Lemma 6.3 If $\left\{a_{1}, a_{2}\right\},\left\{b_{1}, b_{2}\right\}$ are two cocycles in $H^{*}\left(G_{2}\left(\mathbb{C}^{n}\right), \mathcal{Z}\right)$ and

$$
m:=\min \left\{\left(a_{1}-a_{2}\right),\left(b_{1}-b_{2}\right)\right\}
$$

then one has

$$
\left\{a_{1}, a_{2}\right\}\left\{b_{1}, b_{2}\right\}=\sum_{i=0}^{m}\left\{a_{1}+b_{1}-i, a_{2}+b_{2}+i\right\}
$$


Proof: Direct consequence of the Littlewood Richardson rule. (Compare with [13].)

For the following Lemma let $[x]$ denote the largest integer smaller or equal to $x$.

Lemma 6.4 If $\left\{a_{1 l}, a_{2 l}\right\} \in H^{*}\left(G_{2}\left(\mathbb{C}^{n}\right), \mathcal{Z}\right), l=1, \ldots, r$, are $r$ Schubert cocycles with

$$
a_{11}-a_{21} \geq \cdots \geq a_{1 r}-a_{2 r}
$$

and

$$
m:=\min \left\{\left[\frac{1}{2} \sum_{l=1}^{r}\left(a_{1 l}-a_{2 l}\right)\right], \sum_{l=2}^{r}\left(a_{1 l}-a_{2 l}\right)\right\}
$$

then there are positive nonzero integers $c_{i}$ such that

$$
\prod_{l=1}^{r}\left\{a_{1 l}, a_{2 l}\right\}=\sum_{i=0}^{m} c_{i}\left\{\sum_{l=1}^{r} a_{1 l}-i, \sum_{l=1}^{r} a_{2 l}+i\right\} .
$$

In particular if $\sum_{l=1}^{r} a_{1 l} \leq m+n-2$ at least one summand is nonzero and therefore the whole product is nonzero.

Proof: Let $\alpha \in\{2, \ldots, r\}$ be the largest integer with the property that

$$
\left(a_{11}-a_{21}\right) \geq \sum_{l=2}^{\alpha}\left(a_{1 l}-a_{2 l}\right)
$$

Denote with $\tilde{m}:=\sum_{l=2}^{\alpha}\left(a_{1 l}-a_{2 l}\right)$. Using inductively Lemma 6.3 one sees that

$$
\prod_{l=1}^{\alpha}\left\{a_{1 l}, a_{2 l}\right\}=\sum_{i=0}^{\tilde{m}} \tilde{c}_{i}\left\{\sum_{l=1}^{\alpha} a_{1 l}-i, \sum_{l=1}^{\alpha} a_{2 l}+i\right\}
$$

with positive, nonzero constants $\tilde{c}_{i}$. In particular if $\alpha=r$ then $m=\tilde{m}$ and the result is proven. If $\alpha<r$ then $\left(a_{11}-a_{21}\right)<\sum_{l=2}^{r}\left(a_{1 l}-a_{2 l}\right)$ and therefore $m=\left[\frac{1}{2} \sum_{l=1}^{r}\left(a_{1 l}-a_{2 l}\right)\right]$. Multiplying inductively expression (6.9) with the factors $\left\{a_{1 l}, a_{2 l}\right\}, l=\alpha+1, \ldots, r$ one deduces also in this case, using the fact that all Littlewood Richardson coefficients are positive, that $\prod_{l=1}^{r}\left\{a_{1 l}, a_{2 l}\right\}=\sum_{i=0}^{m} c_{i}\left\{x_{i}, y_{i}\right\}$, where

$$
\sum_{l=1}^{r} a_{1 l}-m \leq x_{i} \leq \sum_{l=1}^{r} a_{1 l} \text { and } \sum_{l=1}^{r} a_{2 l} \leq y_{i} \leq \sum_{l=1}^{r} a_{2 l}+m .
$$

In particular, if $\sum_{l=1}^{r} a_{1 l}-m \leq n-2$, the product is nonzero, which completes the proof.

As a direct consequence of this Lemma we obtain a description of all maximal nonzero products in $H^{*}\left(G_{2}\left(\mathbb{C}^{n}\right), \mathcal{Z}\right)$. 
Theorem 6.5 Assume $\left\{a_{1 l}, a_{2 l}\right\} \in H^{*}\left(G_{2}\left(\mathbb{C}^{n}\right), \mathcal{Z}\right), l=1, \ldots, r$, are $r$ cocycles with

$$
\sum_{l=1}^{r}\left(a_{1 l}+a_{2 l}\right)=2(n-2) .
$$

Then $\prod_{l=1}^{r}\left\{a_{1 l}, a_{2 l}\right\} \neq 0$ if, and only if

$$
\left(a_{1 j}-a_{2 j}\right) \leq \sum_{l \in\{1, \ldots, j-1, j+1, \ldots, r\}}\left(a_{1 l}-a_{2 l}\right), \quad j=1, \ldots, r .
$$

Proof: After a possible reindexing we can assume that

$$
a_{11}-a_{21} \geq \cdots \geq a_{1 r}-a_{2 r}
$$

Because of assumption (6.12), $m=\left[\frac{1}{2} \sum_{l=1}^{r}\left(a_{1 l}-a_{2 l}\right)\right]$. Because of the description of $H^{*}\left(G_{2}\left(\mathbb{C}^{n}\right), \mathcal{Z}\right)$ in (3.12) it is clear that the product is nonzero if, and only if the coefficient of $\{n-2, n-2\} \in H^{2(n-2)}\left(G_{2}\left(\mathbb{C}^{n}\right), \mathcal{Z}\right)$ in the product expansion is nonzero. By the last Lemma this is the case iff $\sum_{l=1}^{r} a_{1 l} \leq m+n-2$. Moreover because of (6.11) the number $\frac{1}{2} \sum_{l=1}^{r}\left(a_{1 l}-a_{2 l}\right)$ is an integer. But then $\sum_{l=1}^{r} a_{1 l} \leq m+n-2$ is equivalent to $\sum_{l=1}^{r}\left(a_{1 l}+a_{2 l}\right) \leq 2(n-2)$ which is true by assumption $(6.11)$.

Remark 6.6 If $\sum_{l=1}^{r}\left(a_{1 l}+a_{2 l}\right)<2(n-2)$ then it follows from the last proof that assumption (6.12) is still sufficient for the product $\prod_{l=1}^{r}\left\{a_{1 l}, a_{2 l}\right\}$ to be nonzero.

Combining Theorem 6.5 with Theorem 4.3 one finally has:

Theorem 6.7 Let $\left(i_{1 l}, i_{2 l}\right)$ be $r+1$ pairs of integers with:

$$
\begin{gathered}
1 \leq i_{1 l}<i_{2 l} \leq n, \quad l=1, \ldots, r+1 \\
r(2 n-1)+3 \leq \sum_{l=1}^{r+1}\left(i_{1 l}+i_{2 l}\right) \\
i_{2 j}-i_{1 j} \leq 1-r+\sum_{l \in\{1, \ldots, j-1, j+1, \ldots, r+1\}}\left(i_{2 l}-i_{1 l}\right), \quad j=1, \ldots, r+1 .
\end{gathered}
$$

Then for any set of Hermitian matrices $A_{1}, \ldots, A_{r+1} \in \mathbb{C}^{n \times n}$ satisfying the relation $A_{r+1}=A_{1}+\cdots+A_{r}$ the following eigenvalue inequalities hold:

$$
\begin{aligned}
& \lambda_{n-i_{1, r+1}+1}\left(A_{r+1}\right)+\lambda_{n-i_{2, r+1}+1}\left(A_{r+1}\right) \geq \sum_{l=1}^{r}\left(\lambda_{i_{1 l}}\left(A_{l}\right)+\lambda_{i_{2 l}}\left(A_{l}\right)\right) \\
& \lambda_{i_{1, r+1}}\left(A_{r+1}\right)+\lambda_{i_{2, r+1}}\left(A_{r+1}\right) \leq \sum_{l=1}^{r}\left(\lambda_{n-i_{1 l}+1}\left(A_{l}\right)+\lambda_{n-i_{2 l}+1}\left(A_{l}\right)\right) .
\end{aligned}
$$


Proof: Denote with $a_{1 l}=n-i_{1 l}-1$ and $a_{2 l}=n-i_{2 l}$. Then condition (6.15) is equivalent to the condition $\sum_{l=1}^{r+1}\left(a_{1 l}+a_{2 l}\right) \leq 2(n-2)$ and condition (6.16) is equivalent to inequality (6.12). By Remark 6.6 the product $\prod_{l=1}^{r+1}\left\{n-i_{1 l}-1, n-i_{2 l}\right\}$ is nonzero and the result follows once again from Theorem 4.3.

In order to illustrate the theorem in the case $r=2$, let $A=A_{1}, B=A_{2}$ and let

$$
\begin{aligned}
& \left(i_{1,1}, i_{2,1}\right)=\left(n-a_{2}+1, n-a_{1}+1\right), \\
& \left(i_{1,2}, i_{2,2}\right)=\left(n-b_{2}+1, n-b_{1}+1\right), \\
& \left(i_{1,3}, i_{2,3}\right)=\left(c_{1}, c_{2}\right) .
\end{aligned}
$$

Then we obtain

Corollary 6.8 Let $1 \leq a_{1}<a_{2} \leq n, 1 \leq b_{1}<b_{2} \leq n$ and $1 \leq c_{1}<c_{2} \leq n$ satisfy the system of linear inequalities

$$
\begin{aligned}
a_{1}+a_{2}+b_{1}+b_{2} & \leq c_{1}+c_{2}+3 \\
a_{2}-a_{1} & \leq b_{2}-b_{1}+c_{2}-c_{1}-1 \\
b_{2}-b_{1} & \leq a_{2}-a_{1}+c_{2}-c_{1}-1 \\
c_{2}-c_{1} & \leq a_{2}-a_{1}+b_{2}-b_{1}-1 .
\end{aligned}
$$

Then the eigenvalue inequality

$$
\lambda_{c_{1}}(A+B)+\lambda_{c_{2}}(A+B) \leq \lambda_{a_{1}}(A)+\lambda_{a_{2}}(A)+\lambda_{b_{1}}(B)+\lambda_{b_{2}}(B)
$$

holds for any pair of Hermitian $n \times n$ matrices $A, B$.

We would like to remark that the assumptions in Corollary 6.8 imply the assumptions in Theorem 8 of Horn [7]. In particular it is also possible to derive the inequality (6.26) by the methods developed in [7].

In the last part of this section we describe all maximal nonzero products of $H^{*}\left(G_{k}\left(\mathbb{C}^{n}\right), \mathcal{Z}\right)$ consisting of 3 factors. The results are based on a description of the Littlewood Richardson coefficients as given by Schlosser in [13].

In the following we explain his description and simultaneously adapt the notation for our purposes.

Let $\mu:=\left(\mu_{1}, \ldots, \mu_{k}\right), \nu:=\left(\nu_{1}, \ldots, \nu_{k}\right)$ and $\lambda:=\left(\lambda_{1}, \ldots, \lambda_{k}\right)$ be partitions. We are interested in conditions when the Littlewood Richardson coefficient $c_{\mu, \nu}^{\lambda}$ is nonzero. We will use the combinatorial description of $c_{\mu, \nu}^{\lambda}$ as given in Theorem 3.7 and the following parameterization by Schlosser [13].

Consider the tableau $\lambda$ and denote with $p_{h i}$ the number of boxes in the skew tableau $\lambda / \mu$ with label $i$ in the $h$-th row. This gives us the following description for 
the tableau $\lambda$ :

\begin{tabular}{c|ccccc|c} 
row & & & & & \\
\hline 1 & $\mu_{1}$ & $p_{11}$ & & & & $\lambda_{1}$ \\
2 & $\mu_{2}$ & $p_{21}$ & $p_{22}$ & & & $\lambda_{2}$ \\
$\vdots$ & $\vdots$ & $\vdots$ & $\vdots$ & $\ddots$ & & $\vdots$ \\
$k$ & $\mu_{k}$ & $p_{k 1}$ & $p_{k 2}$ & $\ldots$ & $p_{k k}$ & $\lambda_{k}$ \\
\hline & $|\mu|$ & $\nu_{1}$ & $\nu_{2}$ & $\ldots$ & $\nu_{k}$ & total
\end{tabular}

Of course not all configurations of numbers $p_{h i}$ will result in a filling compatible with the rule of Littlewood and Richardson. On the other hand, as shown in [13], one can iteratively fill the skew tableau $\lambda / \mu$, starting with $p_{k 1}$ and proceeding inductively with

$$
p_{h i}, \quad h=k, \ldots, i+1, \quad i=1, \ldots, k-1,
$$

subject to the following inequalities:

$$
\operatorname{Max}(h, i ;(\nu)) \leq p_{h i} \leq \operatorname{Min}(h, i ;(\nu),(\mu))
$$

where

$$
\begin{gathered}
\operatorname{Max}(h, i ;(\nu)):=\max \left\{0, \nu_{i}-\nu_{i-1}-\sum_{j=h+1}^{k} p_{j i}+\sum_{j=h-1}^{k} p_{j, i-1}\right\} \\
\operatorname{Min}(h, i ;(\nu),(\mu)):=\min \left\{\mu_{h-1}-\mu_{h}+\sum_{j=1}^{i-1}\left(p_{h-1, j}-p_{h, j}\right), \nu_{i}-\sum_{j=h+1}^{k} p_{j i}\right\}
\end{gathered}
$$

and

$$
p_{i i}=\nu_{h}-\sum_{h=i+1}^{k} p_{h i}, i=1, \ldots, k
$$

In this iterative scheme we assume that

$$
\nu_{0}=0, \quad p_{0, j}=0, \quad p_{h, 0}=0 .
$$

For our purposes the main result, which is stated in similar form in [13, Theorem 1], is:

Theorem 6.9 Let $\mu, \nu$ be partitions and let $p_{h i}$ be iteratively described through (6.28) and (6.29). Denote with

$$
\lambda_{h}:=\mu_{h}+\sum_{i=1}^{h} p_{h i}, h=1, \ldots, k .
$$

Then $\lambda:=\left(\lambda_{1}, \ldots, \lambda_{k}\right)$ describes a tableau and the Littlewood Richardson coefficient $c_{\mu, \nu}^{\lambda}$ is nonzero. 
Corollary 6.10 Let $\mu, \nu$ be partitions and let $\lambda$ satisfy the inequalities induced by the iterative scheme (6.28) and (6.29). Then

$$
\{\mu\}\{\nu\}\left\{n-k-\lambda_{k}, \ldots, n-k-\lambda_{1}\right\} \neq 0 .
$$

Proof: The cocycle $\left\{n-k-\lambda_{k}, \ldots, n-k-\lambda_{1}\right\}$ is the Poincaré dual of the cocycle $\{\lambda\}$ and because the Littlewood Richardson coefficient $c_{\mu, \nu}^{\lambda}$ is nonzero the results follows from Lemma 6.1.

Corollary 6.11 Let $A, B$ be complex Hermitian $n \times n$ matrices. Let $\mu, \nu$ be partitions and let $\lambda$ satisfy the inequalities induced by (6.28) and (6.29). Let

$$
\begin{aligned}
a_{1} & :=\mu_{k}+1, \ldots, a_{k}:=\mu_{1}+k \\
b_{1} & :=\nu_{k}+1, \ldots, b_{k}:=\nu_{1}+k \\
c_{1} & :=\lambda_{k}+1, \ldots, c_{k}:=\lambda_{1}+k
\end{aligned}
$$

Then

$$
\sum_{\nu=1}^{k} \lambda_{c_{\nu}}(A+B) \leq \sum_{\nu=1}^{k} \lambda_{a_{\nu}}(A)+\sum_{\nu=1}^{k} \lambda_{b_{\nu}}(B) .
$$

Proof: Direct consequence of equation (6.32) and Theorem 4.3.

Remark 6.12 The inequalities of Freede and Thompson (5.7) are in the following way a special case of this Corollary. Choose in the iterative scheme (6.28) and (6.29) $p_{h i}=0$ for $h \neq i$ and $p_{h h}=\nu_{h}$. Then $\lambda=\mu+\nu$ and the nonzero product (6.32) reduces to the product $(5.8)$.

Acknowledgments. The authors would like to thank the anonymous referee for the careful review of the paper.

\section{References}

[1] R. Bhatia. Perturbation Bounds for Matrix Eigenvalues, volume 162 of Research Notes in Mathematics. Pitman, 1987.

[2] R. W. Brockett and C. I. Byrnes. Multivariable Nyquist criteria, root loci and pole placement: A geometric viewpoint. IEEE Trans. Automat. Control, AC26:271-284, 1981.

[3] W. Fulton. Intersection Theory. Ergebnisse der Mathematik und ihrer Grenzgebiete. Springer Verlag, Berlin, Heidelberg, New York, 1984. 
[4] P. Griffiths and J. Harris. Principles of Algebraic Geometry. John Wiley \& Sons, New York, 1978.

[5] J. Hersch and B. P. Zwahlen. Evaluation par défault pour une somme quelconque de valeurs propres $\gamma_{k}$ d'un opérateur $C=A+B$ à l'aide de valeurs $\alpha_{i}$ de $A$ and $\beta_{i}$ de B. C.R. Acad. Sc. Paris, 254:1559-1561, 1962.

[6] H. Hiller. Geometry of Coxeter Groups, volume 54 of Research Notes in Mathematics. Pitman, 1982.

[7] A. Horn. Eigenvalues of sums of Hermitian matrices. Pacific J. Math, 12:225-241, 1962.

[8] S. Johnson. The Schubert Calculus and Eigenvalue Inequalities for Sums of Hermitian Matrices. PhD thesis, University of California, Santa Barbara, 1979.

[9] B. V. Lidskii. Spectral polhyedron of a sum of two Hermitian matrices. Functional Anal. Appl., 10:139-140, 1982.

[10] I. G. Macdonald. Symmetric Functions and Hall Polynomials. Oxford University Press, Oxford, 1979.

[11] R. C. Riddel. Minimax problems in Grassmann manifolds. Adv. in Math, 54:107199, 1984.

[12] J. Rosenthal. New results in pole assignment by real output feedback. SIAM J. Control Optim., 30(1):203-211, 1992.

[13] H. Schlosser. A closed formula for the rule of Littlewood Richardson with appl. in the theory of repres. of $g l(v)$ and the superalgebra $p l(v)$. Math. Nachr., 151:315326, 1991.

[14] R. Stanley. Some Combinatorial Aspects of the Schubert Calculus, pages 217-251. Lecture Notes in Mathematics \# 579. Springer Verlag, 1977.

[15] R. C. Thompson. The Schubert calculus and matrix spectral inequalities. Linear Algebra Appl., 117:176-179, 1989.

[16] R. C. Thompson. High, low, and quantitative roads in linear algebra. Linear Algebra Appl., 162-164:23-64, 1992.

[17] R. C. Thompson and L. Freede. On the eigenvalues of a sum of Hermitian matrices. Linear Algebra Appl., 4:369-376, 1971.

[18] H. Weyl. Das asymptotische Verteilungsgesetz der Eigenwerte linearer partieller Differentialgleichungen. Math. Ann, 71:441-479, 1912.

[19] H. Wielandt. An extremum property of sums of eigenvalues. Proc. Amer. Math. Soc., 6:106-110, 1955. 